\title{
A Novel Approach to Reduce L2 Miss Latency in Shared-Memory Multiprocessors
}

\author{
Manuel E. Acacio, José González, José M. García \\ Dpto. Ing. y Tecnología de Computadores \\ Universidad de Murcia \\ 30071 Murcia (Spain) \\ \{meacacio, joseg, jmgarcia\}@ditec.um.es
}

\author{
José Duato \\ Dpto. Inf. de Sistemas y Computadores \\ Universidad Politécnica de Valencia \\ 46071 Valencia (Spain) \\ jduato@gap.upv.es
}

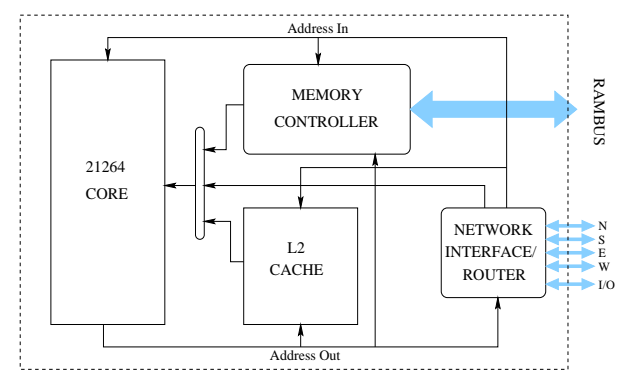

Figure 1. Alpha 21364 chip block diagram

line. However, the implementations of the shared-memory paradigm have limited scalability, then becoming infeasible for very large-scale systems, which use the messagepassing paradigm. Examples of such machines are the ASCI Red, the ASCI Blue Pacific and the ASCI White multiprocessors.

There are two main issues limiting the scalability of ccNUMA designs: the hardware overhead of using directories and the usually long L2 miss latencies. The most important component of the hardware overhead is the amount of memory required to store the directory information, particularly the sharing code. An effective way to reduce this overhead is by using sharing codes whose size (in bits) is not a linear function of the number of nodes, unlike the well-known bit-vector one [5]. Although the use of these compressed directories significantly reduce the memory overhead, it has also negative consequences on performance. These are caused by the appearance of unnecessary coherence messages, that is, coherence messages that would not be sent if a precise sharing code such as bitvector were used. Organizing the directory as a two-level architecture was proposed in our previous work as an effective way to significantly reducing the memory overhead entailed by the directory information while achieving the performance of a non-scalable bit-vector directory [1].

Long miss latencies of directory protocols are caused by the inefficiencies that the distributed nature of the protocols and the underlying scalable network imply. One of such inefficiencies is the indirection introduced by the directory access. The consequences of such indirection are particularly serious for cache-to-cache transfers which, in some cases, represent more than $60 \%$ of the misses [3].

Current technology improvements allow designers to implement some key components of the system inside the processor chip. For example, the Compaq Alpha 21364 [7] includes on-chip memory controller, coherence hardware and network interface and router (see Figure 1). Taking 


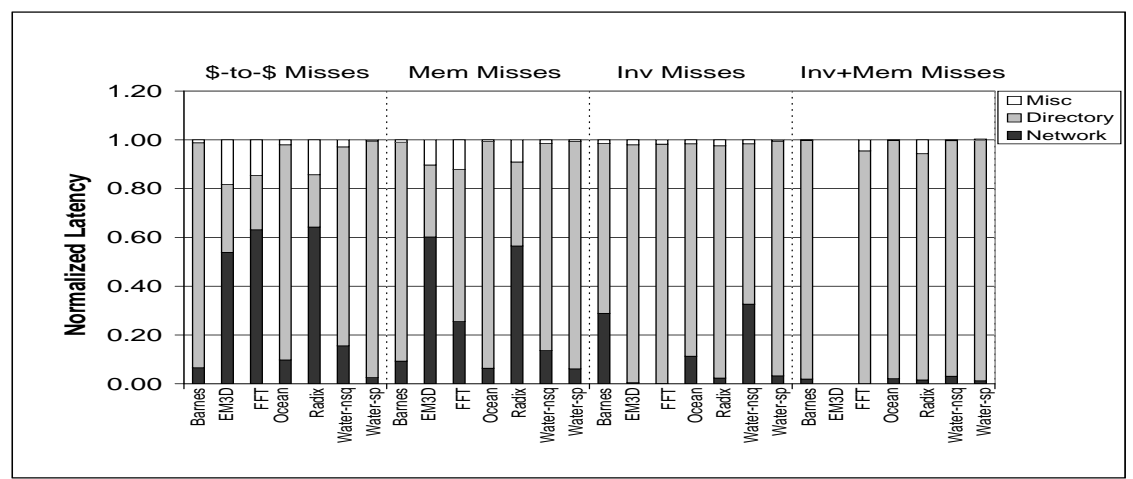

Figure 2. Normalized Average Latency for $\$$-to- $\$, M e m$, Inv and Inv+Mem misses

these system organizations as a starting point and considering the opportunities provided by current integration scale, in this work we propose a novel node organization especially designed to reduce the usually long L2 miss latencies and the memory overhead of using directories that characterize cc-NUMA multiprocessors and limits their scalability. Our proposal replaces the traditional directory with a novel three-level directory architecture and adds a small shared data cache to each of the nodes that form the multiprocessor. The first-level directory, which stores directory information for the most recently referenced memory lines, as well as the small shared data cache, which contains those memory lines that are expected to be requested in a near future, are integrated into the processor chip in every node, whereas second- and third-level directories are placed outside the processor. The on-chip integration of the small first-level directory and the shared data cache enhances performance whereas memory overhead is significantly reduced by having a compressed third level. Now, those cache misses that find their corresponding directory entry and memory line (when needed) in the firstlevel directory and shared data cache, respectively, can be directly served from the processor chip, significantly reducing the time needed by the home directory to satisfy them. Note that, unlike the remote data caches (RDCs) used in some systems (for example, in [11]) to cache lines that are fetched to the node from remote memories, the shared data cache proposed in our design tries to reduce the latency of accessing main memory (when needed) by quickly providing data from the processor itself to the requesting node. This is possible since coherence hardware, memory controller and network router are also included inside the processor die.

The rest of the paper is organized as follows. Section 2 presents a classification of the L 2 misses found in ccNUMA systems. The new node architecture is proposed and justified in Section 3. Section 4 discusses our evaluation methodology. Section 5 shows a detailed performance evaluation of our novel proposal. The related work is presented in Section 6 and Section 7 concludes the paper.

\section{A Taxonomy of the L2 Misses}

Each time an access to a certain memory line (i.e., a load or a store) misses in the $\mathrm{L} 2$ cache, a request is addressed to the home directory of the line. The actions to be undertaken by the home directory depend on the coherence protocol, the access type and the state of the memory line.

In this section we present a taxonomy of the L 2 misses found in cc-NUMA multiprocessors in terms of the actions done by the directory. Assuming that an invalidation-based four-state MESI coherence protocol is used, L2 misses can be classified into the following four categories:

1. Cache-to-cache transfer misses $(\$-$ to- $\$)$ : There is a single processor caching the memory line. In this case, the directory forwards the request to the owner of the line. They are also known as 3-Hop misses.

2. Access to memory misses $(\mathrm{Mem})$ : They appear for write accesses when there is not any processor caching the requested line and for read accesses when the requested line is cached by zero or more than one processor. The home directory satisfies the miss by accessing main memory in order to provide the line.

3. Invalidate misses (Inv): A write access for a memory line comes to the directory, there are several nodes holding a copy of the line and one of them is the processor issuing the access. The directory must invalidate all the copies of the memory line but the one held by the requesting processor.

4. Invalidate and access to memory misses $($ Inv $+M e m)$ : Caused by a write access for which there are several nodes caching the line but none of them is the one issuing the access. Now, the directory must first invalidate all copies of the line. Then, it sends a reply with the memory line to the requesting processor.

\begin{tabular}{|c|c|c|c|c|}
\cline { 2 - 5 } \multicolumn{1}{c|}{} & \multicolumn{4}{c|}{ Directory States } \\
\cline { 2 - 5 } \multicolumn{1}{c|}{} & Private & \multicolumn{2}{|c|}{ Shared } & Uncached \\
\hline \hline Load Miss & $\$$-to- $\$$ & \multicolumn{2}{|c|}{ Mem } & Mem \\
\hline Store Miss & $\$$-to-\$ & $\begin{array}{c}\text { Inv } \\
\text { (Upgrade) }\end{array}$ & Inv + Mem & Mem \\
\hline
\end{tabular}

Table 1. Directory actions to satisfy load/store misses

Table 1 shows the actions that the directory performs to satisfy load and store misses. A load miss is either satisfied by a cache-to-cache transfer or an access to memory. On the other hand, any of the four actions could be used for store misses. For each one of the former categories, Figure 2 presents the normalized average miss latency obtained when running several applications on the base system assumed in this work. Average miss latency is split into network latency, directory latency and miscellaneous latency (buses, cache accesses...). Further details about the evaluation methodology are included in Section 4. As can be observed, the most important fraction of the average miss latency is caused by the directory. This is true for all the applications in the Inv and Inv+Mem cases, whereas only two and three applications found network latency to exceed directory one in the Mem and \$-to-\$ categories, respectively. 


\section{An Approach to Reduce L2 Miss Latencies}

Previous section classified L2 misses in terms of the actions carried out by home directories to satisfy them and identified directory latency to constitute the most important component of the L2 miss latency for the majority of the applications. For $\$$-to- $\$$ and $\mathrm{Mem}$ misses, directory latency is caused by the access to main memory to obtain the corresponding directory entry (for $\mathrm{Mem}$ misses, memory line lookup occurs in parallel with the access to the directory information, as in [10]). For Inv and Inv+Mem misses, directory latency comprises the cycles needed to obtain directory information and to create and send invalidation messages as well as to receive the corresponding acknowledgments (again, for a Inv+Mem miss the directory entry and the requested line are simultaneously obtained).

Assuming that nodes similar to the one presented in Figure 1 are used to form a cc-NUMA multiprocessor (as described in [7]), in this section we propose a novel node organization especially designed to reduce the latency of L2 misses by significantly decreasing the component of the latency caused by the directory. Additionally, our proposal minimizes the memory overhead caused by directory information. For a detailed description refer to [2].

\subsection{Node Architecture}

The proposed node organization adds several elements to the basic node architecture shown in Figure 1. In particular, the directory is organized as a three-level structure and a shared cache for memory lines that are frequently accessed by several nodes is included. The three-level directory architecture consists of:

1. First-level directory: This directory level is located inside the processor chip, close to the directory controller. It is managed as a cache and uses a small set of entries, each one containing a precise sharing code consisting of three pointers (of $\log _{2} N$ bits each one, for a $N$-node system). Note that, as it is shown in [5], a small number of pointers generally suffices to keep track of the nodes caching a memory line.

2. Second-level Directory: It is located outside the processor chip and also has a small number of entries. In this case, a non-scalable but precise bit-vector sharing code is employed. The second-level directory can be seen as a victim cache of the fist level since it contains those entries that have been evicted from the first-level directory or do not fit there due to the total number of sharers is larger than the available number of pointers (three, in our case).

3. Third-level Directory: This level constitutes the complete directory structure (i.e., an entry per memory line) and it is located near main memory (it could be included in main memory). Each entry in this level uses a compressed sharing code to drastically reduce memory requirements. In particular, we use the $\mathrm{Bi}$ nary Tree with Subtrees $(B T-S u T)$ [1] sharing code, which has space complexity $\mathrm{O}\left(\log _{2}(N)\right)$, for a $N$ node system. Sharing information in this level is always updated when changes in the first- or secondlevel directories are performed.

Accesses to the third-level directory imply main memory latency. First-level directory has the latency of a fast on-chip cache whereas the second-level one provides data

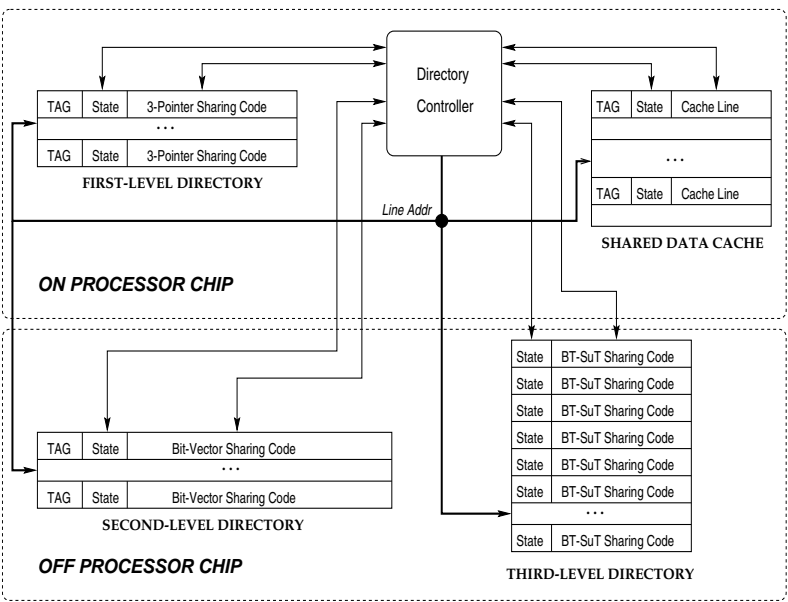

Figure 3. Proposed node architecture

at the same speed as an off-chip cache. This way, \$-to$\$, I n v$ and $I n v+M e m$ misses would be significantly accelerated if their corresponding directory entry were found at the first- or second-level directories, since the directory controller could quickly service them. Due to the locality exhibited by memory references, we expect the firstand second-level directories to satisfy most of the requests, even remote accesses to a home node. Thus, this will bring important reductions in the component of the miss latency owed to the directory.

On the contrary, Mem misses can not take full advantage of finding directory information in the first- or secondlevel directories. For this kind of misses, the directory controller must directly provide the line from main memory. In order to also accelerate $\mathrm{Mem}$ misses, our design includes a small cache inside the processor chip, the shared data cache. This cache stores a copy of those memory lines with a valid copy in main memory (that is, they are in the Shared or Uncached states) and that are expected to be accessed in a near future. As coherence hardware, memory controller and network router are already included inside the processor chip, Mem misses can take significant advantage of finding their corresponding memory line in the shared data cache. State bits are also included in each entry of this shared data cache. The reason for this will be explained in next section.

Figure 3 summarizes the node architecture proposed in this work. This node organization is divided into two different parts (on-chip and off-chip parts) according to their location in the processor chip. The on-chip part includes the first-level directory and the shared data cache. The offchip structure comprises the second- and third-level directories. Tag information is stored in the first- and secondlevel directories as well as in the shared data cache in order to determine whether there is a hit.

\subsection{Directory Controller Operation}

Each time a L2 miss for a certain memory line arrives at the directory controller, the address of the line associated with the request is sent to each one of the directory levels as well as to the shared data cache and main memory. One of the following situations can take place:

1. The directory entry is found in the first-level directory. In this case the directory controller obtains precise sharing information, ending the access to the second and third levels. For Mem and Inv+Mem misses, 


\begin{tabular}{|c|c|c|c|c|c|}
\hline \multirow{2}{*}{\multicolumn{2}{|c|}{$\begin{array}{c}\text { Directory } \\
\text { Receives }\end{array}$}} & \multicolumn{4}{|c|}{ Directory information found in } \\
\hline & & $\begin{array}{l}\text { First-level directory } \\
\text { (DC1) }\end{array}$ & $\begin{array}{c}\text { Second-level directory } \\
\text { (DC2) }\end{array}$ & $\begin{array}{c}\text { Third-level directory } \\
\text { (MEM) }\end{array}$ & $\begin{array}{l}\text { Shared data cache } \\
\text { (SDC) }\end{array}$ \\
\hline \multirow{4}{*}{$\begin{array}{c}\text { L2 } \\
\text { Miss }\end{array}$} & Miss Type & & & & \\
\hline & \$-to-\$ & Update entry & Move entry to DC1 & Allocate an entry in DC1 & Not allowed \\
\hline & Mem & $\begin{array}{l}\text { Update entry (Sharers } \leq 3 \text { ) } \\
\text { or move entry to DC } 2 \\
\text { Insert line in SDC (if needed) }\end{array}$ & $\begin{array}{l}\text { Update entry (Sharers }>3 \text { ) } \\
\text { or move entry to DC } 1 \\
\text { Insert line in SDC (if needed) }\end{array}$ & $\begin{array}{l}\text { Allocate an entry in DCI } \\
\quad \text { (if line state = Uncached })\end{array}$ & $\begin{array}{l}\text { Allocate an entry in } \\
\text { DC1 and extract line } \\
\text { from SDC }\end{array}$ \\
\hline & $\begin{array}{l}\text { Inv } \\
\text { Inv+Mem }\end{array}$ & $\begin{array}{l}\text { Update entry and } \\
\text { extract line from SDC } \\
\text { (if found) }\end{array}$ & $\begin{array}{l}\text { Move entry to DCI and } \\
\text { extract line from SDC } \\
\text { (if found) }\end{array}$ & Allocate an entry in DCI & Not allowed \\
\hline \multicolumn{2}{|c|}{ Write-back } & $\begin{array}{c}\text { Free DC1 entry } \\
\text { and insert line in SDC }\end{array}$ & $\begin{array}{c}\text { Free DC2 entry } \\
\text { and insert line in SDC }\end{array}$ & Insert line in SDC & Not allowed \\
\hline \multicolumn{2}{|c|}{ \$-to-\$ Response } & $\begin{array}{l}\text { Insert line in SDC } \\
\text { (if load miss) }\end{array}$ & $\begin{array}{c}\text { Insert line in SDC } \\
\text { (if load miss) }\end{array}$ & Nothing & Not allowed \\
\hline
\end{tabular}

Table 2. Directory controller operation

the access to main memory is ended if the memory line is found in the shared data cache. In all cases, the directory controller properly updates sharing information in the first- and third-level directories.

2. The directory entry is found in the second-level directory. Again, precise sharing information is obtained. The directory controller ends the access to the third-level directory whereas the access to the shared data cache has already been completed. Now the acces to main memory is canceled when the memory line was obtained from the shared data cache or it is not needed. Sharing information in the second and third levels is also updated.

3. The directory entry is only found in the third-level directory. The third-level directory provides imprecise but correct sharing information.

On a L2 miss for which an entry in the first-level directory was not found, an entry in this directory level is allocated only when precise sharing information can be guaranteed. An entry in the shared data cache is allocated in one of these three situations:

1. On a Mem miss for which an entry is present in the first- or second-level directories.

2. On a write-back message from the owner node of the line. This message is caused by a replacement from the single L2 cache holding the memory line and always includes a copy of the line ${ }^{1}$. Since no sharing code is necessary, the state bits of the associated entry in the shared data cache are used to codify the state of the line. This way an entry in the first- or second-level directories will not be wasted.

3. On the response to a $\$$-to- $\$$ miss indicating that a cache-to-cache transfer was performed, when the miss was caused by a load instruction. The state of the line was changed from Private to Shared, and the acknowledgment message contains a copy of the line ${ }^{1}$.

An entry in the first- and second-level directories is freed each time a write-back message for a memory line in the Private state is received. An entry in the shared data cache is freed in two cases: first, when a processor obtains an exclusive copy of the memory line, and, second, when its associated directory entry is evicted from the second-level directory.

Replacements from the second-level directory are discarded, since correct information is ensured to be present in

\footnotetext{
${ }^{1}$ The original coherence protocol did not include a copy of the line neither in a write-back message nor in a cache-to-cache transfer response when the copy contained in main memory was valid.
}

the third-level directory. The same occurs for those memory lines that are evicted from the shared data cache, since main memory contains a valid copy of them. Finally, replacements in the first- and second-level directories are not allowed for entries associated to memory lines with pending coherence transactions. Table 2 summarizes the most important issues described in this section.

\subsection{Implementation Issues}

In this work we assume the organization of the first- and second-level directory caches as well as of the shared data cache to be fully associative, with a LRU replacement policy $^{2}$. Each line in the first- and second-level directories contains a single directory entry. Three states are possible for a line in the shared data cache: not present, present in the Uncached state or present in the Shared state.

We assume the directory controller to provide write buffers to update the three directory levels, the shared data cache and main memory. Thus, writes to each one of the directory levels as well as to main memory and to the shared data cache are assumed to occur immediately.

Finally, the first- and second-level directories are implemented as directory caches, using the usual technologies for on-chip and off-chip processor caches, respectively. The small sharing code used for the third-level directory would avoid the need of external storage for this directory level since, as in [4], it could be directly stored in main memory by computing ECC at a coarser granularity and utilizing the unused bits.

\section{Simulation Environment}

We have used a modified version of Rice Simulator for ILP Multiprocessors (RSIM), a detailed execution-driven simulator [14]. The modeled system is a cc-NUMA with 64 uniprocessor nodes that implements an invalidation-based, four-state MESI directory-based cache coherence protocol and sequential consistency. Table 3 summarizes the parameters of the simulated system. These values have been chosen to be similar to the parameters of current multiprocessors.

In order to evaluate the benefits of our proposals, we have selected several scientific applications covering a variety of computation and communication patterns. Barnes (8,192 Bodies,4 timesteps), FFT (256K complex doubles), Ocean $(258 \times 258$ grid), Radix (2M keys, 1024 radix), Water-Spatial (512 molecules,4 timesteps) and Water-Nsquared (512 molecules, 4 timesteps) are from the SPLASH-2 benchmark suite [16]. EM3D (38,400 nodes,

\footnotetext{
${ }^{2}$ Practical implementations can be set-associative, achieving similar performance at lower cost [12].
} 


\begin{tabular}{|l|l|}
\hline \multicolumn{2}{|c|}{ 64-Node System } \\
\hline \hline ILP Processor Speed & $1 \mathrm{GHz}$ \\
\hline Cache line size & 64 bytes \\
L1 cache WT & Direct mapped, 32KB \\
L1 hit time & 2 cycles \\
L2 cache WB & 4 -way associative, 512KB \\
L2 hit time & 15 cycles, pipelined \\
\hline Directory controller cycle (on-chip) & 1 cycle \\
$1^{\text {st }}$-level directory access time (on-chip) & 1 cycle \\
$2^{\text {nd }}$-level directory access time (off-chip) & 10 cycles \\
$3^{\text {rd } \text {-level directory access time (off-chip) }}$ & 70 cycles \\
\hline Shared data cache access time (on-chip) & 6 cycles \\
Memory access time & 70 cycles (70 ns) \\
\hline Bus speed/width & $1 \mathrm{GHz} / 8$ bytes \\
\hline Network Topology & 2 -dimensional mesh \\
Flit size & 8 bytes \\
Non-data message size & 2 Flits \\
Router speed & $250 \mathrm{MHz}$ \\
Channel speed & $500 \mathrm{MHz}$ \\
Channel width & 32 bits \\
\hline
\end{tabular}

Table 3. Base system parameters

25 time steps) is a shared-memory implementation of the Split-C benchmark. All experimental results reported in this paper are for the parallel phase of these applications.

Using these applications we compare, through extensive simulation runs, three configurations: the Base system (directory information at main memory) and two configurations using the node architecture presented in Section 3. The first one ( $U C$ system), which gives us the potential of our proposal, uses an unlimited number of entries in the first- and second-level directories as well as in the shared data cache. The second one ( $L C$ system) limits the number of entries in these structures. The directory controller is assumed to be included inside the processor chip as shown in Table 3. Base system uses bit-vector as the sharing code for its single level directory, which obtains the best results since unnecessary coherence messages degrading performance do not appear. As in [1], the coherence protocol used in the $U C$ and $L C$ configurations has been extended to support the use of a compressed third-level directory and also to include always the memory line in write-back messages and in cache-to-cache transfer responses (which increases the number of cycles needed by these messages to reach the corresponding home directory). On the contrary, the Base configuration does not include these overheads.

\section{Simulation Results and Analysis}

In this section we present and analyze simulation results for the Base and $U C$ systems as well as for two instances of the $L C$ configuration. The first one, $L C$ - 1 , limits the number of entries used in the first- and second-level directories and in the shared data cache to 512, 256, and 512, respectively. This results in total sizes of less than 2 and $3 \mathrm{~KB}$ for the first- and second-level directories, respectively, and of $32 \mathrm{~KB}$ for the shared data cache. The other instance, $L C$-2, increases the number of entries for all the components to 1024 , resulting in total sizes of $3 \mathrm{~KB}, 10 \mathrm{~KB}$ and $64 \mathrm{~KB}$ for the first-level directory, second-level directory and shared data cache, respectively. In all cases, the sizes of these components represent a small percentage of the L2 size (a $12.5 \%$ in the worst case).

\subsection{Impact on L2 Miss Latencies}

This section analyzes how the node architecture presented in this work impacts on the latency of L2 misses.

Tables 4 to 7 show the directory structures involved when satisfying each miss type for the $L C-1, L C-2$ and $U C$ configurations. The first and second columns (DCI and $D C 2$ ) present the percentage of misses for which directory information is obtained from the first- and second-level directories, respectively (and, for Mem misses, the cache line from the shared data cache $(S D C)$ ). Thus, these misses would benefit from the novel node architecture proposed in this work, contrary to the ones shown in the column $M E M$, for which main memory must be accessed. Note that all the accesses in the Base configuration are to the main memory.

Figures 4 to 7 illustrate the normalized average latency for each miss type split into network latency, directory latency and miscellaneous latency (buses, cache accesses...), for the Base, $U C, L C-1$ and $L C$ - 2 configurations. Normalized average latencies are computed dividing the average latencies for each one of the configurations by the average latencies for the Base case.

\section{Impact on \$-to-\$ Miss Latencies}

As shown in Figure 4, the small number of entries used in the $L C$ - 1 case for the directory caches as well as for the shared data cache suffices to virtually reach the latency reductions found in the $U C$ case for Barnes $(66 \%)$, EM3D (25\%), Ocean (42\%), Water-nsq (62\%) and Watersp (58\%). As derived from Table 4, for FFT and Radix, more than $80 \%$ of the misses requiring a cache-to-cache transfer need the third-level directory to provide the sharing information. The increased number of entries used in the $L C-2$ case significantly reduces this percentage. Now, only $18.10 \%$ for FFT and $36.88 \%$ for Radix of the $\$$-to$\$$ misses must wait during main memory latency to obtain the sharing information and reductions of $14 \%$ for FFT and $11 \%$ for Radix in average latency are observed. Note that obtaining the directory information from the third-level directory can entail two negative effects on performance: first, the miss must wait until main memory provides the corresponding directory entry and, second, in some situations unnecessary coherence messages could appear as a consequence of the compressed nature of the third-level directory. This second effect can not actually take place for $\$$-to- $\$$ misses since the compressed sharing code used by the third-level directory is wide enough to exactly codify the identity of a single sharer.

\section{Impact on Mem Miss Latencies}

Figure 5 shows the normalized average latencies for Mem misses. For this kind of misses unnecessary coherence messages can not appear. Latency reductions of $45 \%$ for Barnes, $26 \%$ for EM3D, 32\% for Ocean, 37\% for Water-nsq and $40 \%$ for Water-sp are obtained for the $L C-1$ configuration. The reason for these significant reductions is that a large number of the Mem misses found the memory line in the shared data cache and the sharing information in one of the two first directory levels, saving the access to the slower main memory (see Table 5). Again, the former values are very similar to those reached with the $U C$ configuration. For FFT, the $L C-1$ configuration is unable to reduce the latency of Mem misses since more than $93 \%$ of them must obtain the data from main memory. When moving to the $L C-2$ configuration, this percentage decreases and a reduction of $28 \%$ is obtained. However, this reduction still remains far from the potential found for the $U C$ configuration (52\%). Finally, small latency reductions are obtained for Radix when the $U C$ configuration is used (only a $10 \%$ ). As shown in Table 5, a large percentage of the Mem misses suffered by this application $(62.96 \%)$ are for lines that are accessed for the first time, which prevents a shared data cache with an unlimited number of entries from being able to provide the memory line. The percentage of $\mathrm{Mem}$ 


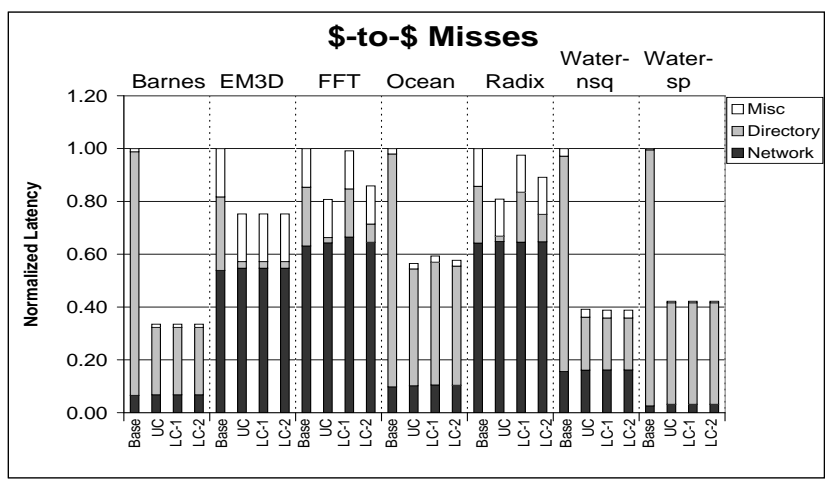

Figure 4. Average $\$$-to- $\$$ miss latency

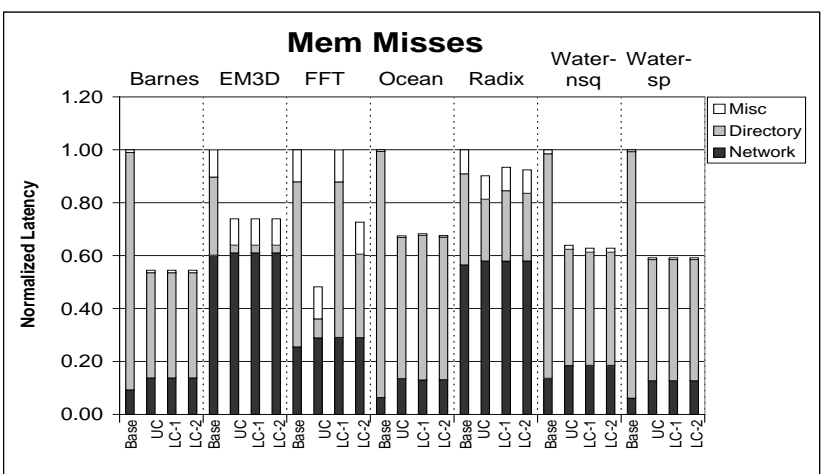

Figure 5. Average $\mathrm{Mem}$ miss latency

\begin{tabular}{|c||c|c|c||c|c|c||c|c|c||}
\cline { 2 - 10 } \multicolumn{1}{c|}{} & \multicolumn{2}{c|}{ LC-1 Configuration } & \multicolumn{2}{c||}{ LC-2 Configuration } & \multicolumn{3}{c||}{ UC Configuration } \\
\hline Application & $D C 1$ & $D C 2$ & $M E M$ & $D C 1$ & $D C 2$ & $M E M$ & $D C 1$ & $D C 2$ & $M E M$ \\
\hline \hline Barnes-Hut & $100.00 \%$ & $0.00 \%$ & $0.00 \%$ & $100.00 \%$ & $0.00 \%$ & $0.00 \%$ & $100.00 \%$ & $0.00 \%$ & $0.00 \%$ \\
\hline EM3D & $100.00 \%$ & $0.00 \%$ & $0.00 \%$ & $100.00 \%$ & $0.00 \%$ & $0.00 \%$ & $100.00 \%$ & $0.00 \%$ & $0.00 \%$ \\
\hline FFT & $11.11 \%$ & $9.68 \%$ & $79.21 \%$ & $35.70 \%$ & $46.20 \%$ & $18.10 \%$ & $100.00 \%$ & $0.00 \%$ & $0.00 \%$ \\
\hline Ocean & $81.66 \%$ & $5.18 \%$ & $13.16 \%$ & $95.60 \%$ & $3.15 \%$ & $1.25 \%$ & $100.00 \%$ & $0.00 \%$ & $0.00 \%$ \\
\hline Radix & $10.65 \%$ & $3.03 \%$ & $86.32 \%$ & $17.86 \%$ & $45.26 \%$ & $36.88 \%$ & $100.00 \%$ & $0.00 \%$ & $0.00 \%$ \\
\hline Water-nsq & $100.00 \%$ & $0.00 \%$ & $0.00 \%$ & $100.00 \%$ & $0.00 \%$ & $0.00 \%$ & $100.00 \%$ & $0.00 \%$ & $0.00 \%$ \\
\hline Water-sp & $100.00 \%$ & $0.00 \%$ & $0.00 \%$ & $100.00 \%$ & $0.00 \%$ & $0.00 \%$ & $100.00 \%$ & $0.00 \%$ & $0.00 \%$ \\
\hline
\end{tabular}

Table 4. How $\$$-to- $\$$ misses are satisfied

\begin{tabular}{|c||c|c|c||c|c|c||c|c|c||}
\cline { 2 - 10 } \multicolumn{1}{c|}{} & \multicolumn{3}{c|}{ LC-1 Configuration } & \multicolumn{3}{c||}{ LC-2 Configuration } & \multicolumn{3}{c||}{ UC Configuration } \\
\hline Application & $\begin{array}{c}D C 1 \\
+S D C\end{array}$ & $\begin{array}{c}D C 2 \\
+S D C\end{array}$ & $M E M$ & $\begin{array}{c}D C 1 \\
+S D C\end{array}$ & $\begin{array}{c}D C 2 \\
+S D C\end{array}$ & $M E M$ & $\begin{array}{c}D C 1 \\
+S D C\end{array}$ & $\begin{array}{c}D C 2 \\
+S D C\end{array}$ & $M E M$ \\
\hline \hline Barnes-Hut & $32.22 \%$ & $67.38 \%$ & $0.40 \%$ & $32.22 \%$ & $67.38 \%$ & $0.40 \%$ & $32.22 \%$ & $67.38 \%$ & $0.40 \%$ \\
\hline EM3D & $96.80 \%$ & $3.20 \%$ & $0.00 \%$ & $96.80 \%$ & $3.20 \%$ & $0.00 \%$ & $96.80 \%$ & $3.20 \%$ & $0.00 \%$ \\
\hline FFT & $0.04 \%$ & $6.39 \%$ & $93.57 \%$ & $0.04 \%$ & $33.20 \%$ & $66.76 \%$ & $0.08 \%$ & $99.92 \%$ & $0.00 \%$ \\
\hline Ocean & $32.35 \%$ & $48.26 \%$ & $19.39 \%$ & $32.66 \%$ & $48.51 \%$ & $18.83 \%$ & $34.06 \%$ & $50.43 \%$ & $15.51 \%$ \\
\hline Radix & $2.04 \%$ & $23.75 \%$ & $74.21 \%$ & $2.05 \%$ & $27.63 \%$ & $70.32 \%$ & $3.26 \%$ & $33.78 \%$ & $62.96 \%$ \\
\hline Water-nsq & $9.44 \%$ & $90.52 \%$ & $0.04 \%$ & $9.44 \%$ & $90.52 \%$ & $0.04 \%$ & $9.59 \%$ & $90.37 \%$ & $0.04 \%$ \\
\hline Water-sp & $10.39 \%$ & $89.58 \%$ & $0.03 \%$ & $10.39 \%$ & $89.58 \%$ & $0.03 \%$ & $10.39 \%$ & $89.58 \%$ & $0.03 \%$ \\
\hline
\end{tabular}

Table 5. How Mem misses are satisfied

\begin{tabular}{|c||c|c|c||c|c|c||c|c|c||}
\cline { 2 - 11 } \multicolumn{1}{c|}{} & \multicolumn{3}{c|}{ LC-1 Configuration } & \multicolumn{2}{c|}{ LC-2 Configuration } & \multicolumn{3}{c||}{ UC Configuration } \\
\hline Application & $D C 1$ & $D C 2$ & $M E M$ & $D C 1$ & $D C 2$ & $M E M$ & $D C 1$ & $D C 2$ & $M E M$ \\
\hline \hline Barnes-Hut & $64.85 \%$ & $35.15 \%$ & $0.00 \%$ & $64.85 \%$ & $35.15 \%$ & $0.00 \%$ & $64.85 \%$ & $35.15 \%$ & $0.00 \%$ \\
\hline EM3D & $75.43 \%$ & $24.57 \%$ & $0.00 \%$ & $75.43 \%$ & $24.57 \%$ & $0.00 \%$ & $75.43 \%$ & $24.57 \%$ & $0.00 \%$ \\
\hline FFT & $22.55 \%$ & $12.20 \%$ & $65.25 \%$ & $47.47 \%$ & $52.08 \%$ & $0.45 \%$ & $100.00 \%$ & $0.00 \%$ & $0.00 \%$ \\
\hline Ocean & $83.12 \%$ & $9.38 \%$ & $7.50 \%$ & $91.70 \%$ & $6.94 \%$ & $1.36 \%$ & $93.79 \%$ & $6.21 \%$ & $0.00 \%$ \\
\hline Radix & $73.14 \%$ & $0.11 \%$ & $26.75 \%$ & $73.18 \%$ & $0.09 \%$ & $26.73 \%$ & $92.57 \%$ & $7.43 \%$ & $0.00 \%$ \\
\hline Water-nsq & $96.73 \%$ & $3.27 \%$ & $0.00 \%$ & $96.73 \%$ & $3.27 \%$ & $0.00 \%$ & $96.73 \%$ & $3.27 \%$ & $0.00 \%$ \\
\hline Water-sp & $51.14 \%$ & $48.86 \%$ & $0.00 \%$ & $51.14 \%$ & $48.86 \%$ & $0.00 \%$ & $51.14 \%$ & $48.86 \%$ & $0.00 \%$ \\
\hline
\end{tabular}

Table 6. How Inv misses are satisfied

\begin{tabular}{|c||c|c|c||c|c|c||c|c|c||}
\cline { 2 - 10 } \multicolumn{1}{c|}{} & \multicolumn{3}{c|}{ LC-1 Configuration } & \multicolumn{2}{c||}{ LC-2 Configuration } & \multicolumn{3}{c||}{ UC Configuration } \\
\hline Application & $D C 1$ & $D C 2$ & $M E M$ & $D C 1$ & $D C 2$ & $M E M$ & $D C 1$ & $D C 2$ & $M E M$ \\
\hline \hline Barnes-Hut & $52.80 \%$ & $47.20 \%$ & $0.00 \%$ & $52.80 \%$ & $47.20 \%$ & $0.00 \%$ & $52.80 \%$ & $47.20 \%$ & $0.00 \%$ \\
\hline EM3D & $0.00 \%$ & $0.00 \%$ & $0.00 \%$ & $0.00 \%$ & $0.00 \%$ & $0.00 \%$ & $0.00 \%$ & $0.00 \%$ & $0.00 \%$ \\
\hline FFT & $14.77 \%$ & $14.90 \%$ & $70.33 \%$ & $44.93 \%$ & $54.79 \%$ & $0.28 \%$ & $100.00 \%$ & $0.00 \%$ & $0.00 \%$ \\
\hline Ocean & $56.69 \%$ & $39.97 \%$ & $3.34 \%$ & $59.27 \%$ & $40.00 \%$ & $0.73 \%$ & $60.49 \%$ & $39.51 \%$ & $0.00 \%$ \\
\hline Radix & $3.18 \%$ & $0.00 \%$ & $96.82 \%$ & $3.18 \%$ & $0.00 \%$ & $96.82 \%$ & $79.24 \%$ & $20.76 \%$ & $0.00 \%$ \\
\hline Water-nsq & $54.99 \%$ & $45.01 \%$ & $0.00 \%$ & $54.99 \%$ & $45.01 \%$ & $0.00 \%$ & $57.06 \%$ & $42.94 \%$ & $0.00 \%$ \\
\hline Water-sp & $39.29 \%$ & $60.71 \%$ & $0.00 \%$ & $39.29 \%$ & $60.71 \%$ & $0.00 \%$ & $39.29 \%$ & $60.71 \%$ & $0.00 \%$ \\
\hline
\end{tabular}

Table 7. How Inv+Mem misses are satisfied

misses satisfied by main memory is even higher for $L C-1$ and $L C-2$ configurations, and latency reductions of $7 \%$ and $8 \%$, respectively, are observed.

\section{Impact on Inv and Inv+Mem Miss Latencies}

Figure 6 shows the normalized average latency for Inv misses. In this case, the directory must send invalidation messages and receive their corresponding acks. Therefore, the main component of the latency is caused by the directory. For Inv misses, $L C-1$ and $U C$ configurations obtain virtually identical latency reductions for Barnes (20\%), EM3D (26\%), Water-nsq (15\%) and Water-sp (45\%). As observed in Table 6, 26\% of the Inv misses in the $L C-1$ and 


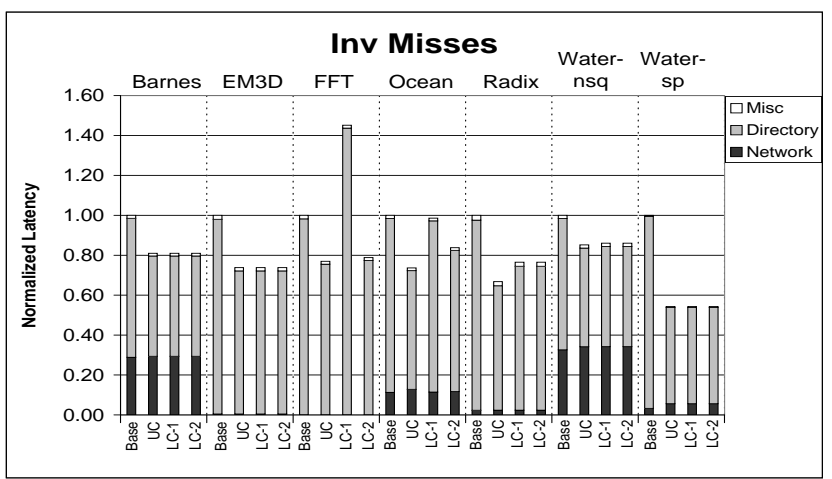

Figure 6. Average Inv miss latency

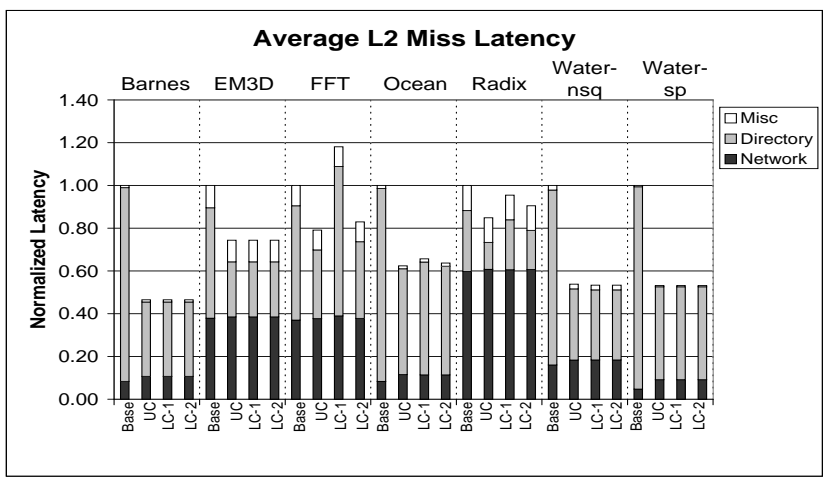

Figure 8. Average L2 miss latency

$L C-2$ configurations must obtain sharing information from main memory for Radix application. In this case, the compressed sharing code used in the third-level directory does not increase the number of invalidation messages observed for the Base case, which explains the small difference between the reduction obtained for the $U C$ configuration and the ones obtained for both the $L C-1$ and the $L C$-2 cases (only 10\%). For FFT, again, an important fraction of the Inv misses found the directory information in main memory when the $L C-1$ configuration was used $(65.25 \%)$. However, in this case, the compression of the third-level directory implies a loss of precision that increases the number of invalidation messages for each invalidation event when comparing with the Base system and, consequently, the average time needed to satisfy this kind of misses. This explains the significant latency increment (more than 40\%) shown in Figure 6. This degradation practically disappears when the number of entries in the first- and second-level directories is increased and the $L C$-2 configuration can obtain the latency reduction of $23 \%$ observed for the $U C$ case. A similar situation also appears for Ocean. In this case, the latency reduction obtained with the $U C$ configuration (26\%) can not be reached when using the $L C-2$ one (17\%).

Figure 7 illustrates the normalized average latency for Inv+Mem misses. When using the $L C-1$ configuration, important latency reductions are also found for Inv+Mem misses for Barnes (50\%), Ocean (22\%), Water-nsq (32\%) and Water-sp (55\%), which coincide with the ones obtained for the $U C$ case. As shown in Table 7, this kind of miss was not found in EM3D application. As in the Inv misses case, the use of the imprecise third-level directory increases the latency of Inv+Mem misses for FFT (more than 40\%) and Radix (20\%) when the $L C-1$ configuration is used. This degradation is completely eliminated in FFT with the use of the $L C-2$ configuration and the performance of the $U C$ case is obtained. However, when moving to the $L C-2$ con-

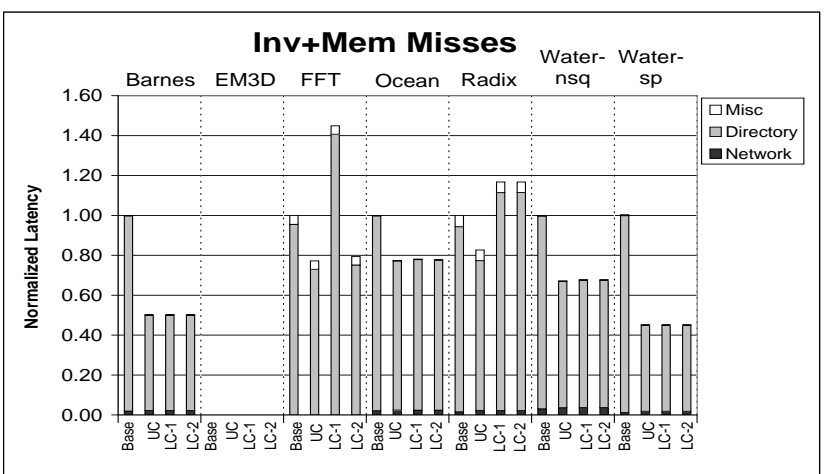

Figure 7. Average Inv+Mem miss latency

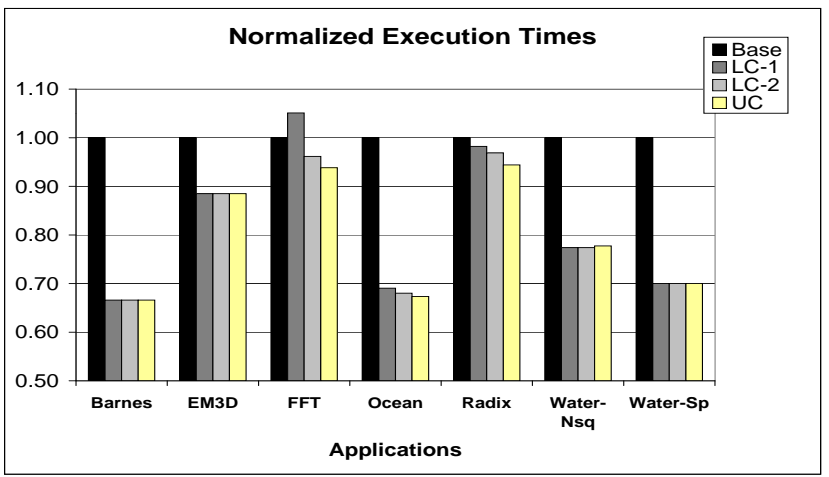

Figure 9. Normalized execution time

figuration in Radix the situation does not improve and a $96.82 \%$ of the Inv+Mem misses must still obtain directory information from main memory.

\subsection{Impact on Execution Time}

For the applications used in our study, Table 8 shows the percentage of L2 misses belonging to each type, whereas Figures 8 and 9 present the normalized average L2 miss latency and execution time, respectively, for each one of the configurations considered in this work.

\begin{tabular}{|c|c|c|c|c|}
\hline Application & \$-to-\$ & Mem & Inv & Inv+Mem \\
\hline \hline Barnes-Hut & $26.43 \%$ & $55.04 \%$ & $17.02 \%$ & $1.51 \%$ \\
\hline EM3D & $34.07 \%$ & $31.86 \%$ & $34.07 \%$ & $0.00 \%$ \\
\hline FFT & $54.12 \%$ & $0.74 \%$ & $37.22 \%$ & $7.92 \%$ \\
\hline Ocean & $42.54 \%$ & $23.39 \%$ & $33.65 \%$ & $0.53 \%$ \\
\hline Radix & $40.81 \%$ & $57.14 \%$ & $1.72 \%$ & $0.33 \%$ \\
\hline Water-nsq & $39.08 \%$ & $34.62 \%$ & $25.74 \%$ & $0.57 \%$ \\
\hline Water-sp & $12.45 \%$ & $82.33 \%$ & $3.64 \%$ & $1.58 \%$ \\
\hline
\end{tabular}

Table 8. L2 misses according to directory actions

Important average L2 miss latency reductions are obtained for those applications that can take significant advantage of the on-chip integration of the first-level directory and the shared date cache. L2 misses have been significantly accelerated in Barnes, Ocean, Water-nsq and Water$\mathrm{sp}$, which motivates the important reductions on the average L2 miss latency observed for these applications (56\% for Barnes, 36\% for Ocean, $46 \%$ for Water-nsq and $47 \%$ for Water-sp). These reductions finally translate into significant improvements in terms of execution time (reductions of 34\% for Barnes, 31\% for Ocean, 22\% for Water-nsq and $30 \%$ for Water-sp). More modest reductions on L2 miss latencies were found for EM3D and a reduction of $25 \%$ on average $\mathrm{L} 2$ miss latency is obtained, resulting in a reduction of $11 \%$ on execution time. For FFT application, the performance degradation observed for Inv and Inv+Mem misses 
when using the $L C-1$ configuration disappears when moving to the $L C-2$ configuration and reductions on average L2 miss latency and execution time close to those reached with the $U C$ configuration are obtained. Finally, only Inv misses could be significantly accelerated for Radix application. However, as shown in Table 8 , only $1.72 \%$ of the misses belong to this category.

\section{Related Work}

In order to significantly reduce the memory overhead entailed by the directory while achieving the same performance as a non-scalable bit-vector directory, we proposed in [1] a two-level directory architecture, which combined a small first-level directory (a few bit-vector entries for the most recently accessed lines) with a compressed secondlevel directory, with one entry per memory line.

Some previously proposed designs, such as the Compaq Alpha 21364 [7] or the Compaq Piranha CMP [4], already include the coherence hardware inside the processor die. However, in such designs, directory information is stored in main memory, which puts main memory latency into the critical path of L2 misses.

Caching directory information was originally proposed in [6] and [13] as a means to reduce the memory overhead entailed by directories. More recently, directory caches have also been used to reduce directory access times [9][12]. In addition, remote data caches (RDCs) have also been used in several designs (as [11]) to accelerate the access to remote data. A RDC caches remote data in local memory resources acting as backup for the processor caches. Multiprocessor-on-a-chip is emerging as an active research topic nowadays. Examples of such architectures can be found in [4][8]. Finally, Torrellas et al. [15] explore how a cache-coherent DSM machine built around Processor-In-Memory chips might be cost-effectively organized.

\section{Conclusions}

We take advantage of current technology trends proposing and studying in this work a novel node architecture especially designed to reduce the long L 2 miss latency by significantly decreasing the component of the latency caused by the directory. Additionally, our approach minimizes the memory overhead caused by directory information.

Our proposal replaces the traditional directory with a novel three-level directory architecture and adds a small shared data cache to each one of the nodes that form the multiprocessor. The first-level directory as well as the small shared data cache are integrated into the processor chip of every node, whereas the second- and third-level directories are placed outside the processor. The on-chip integration of the small first-level directory (which uses a limited number of pointers as sharing code) and the shared data cache ensures performance. The third-level directory is a complete directory structure (one entry per memory line) that uses our BT-SuT compressed sharing code to drastically reduce memory requirements and the second-level directory is a small directory cache (using bit-vector sharing code) that tries to minimize the negative effects of having an imprecise third level as well as to quickly provide directory information when it is not present in the first level.

In order to better understand the reasons for performance improvement, a taxonomy of the L2 misses, according to the actions performed by the directory to satisfy them, has been presented. Latency reductions up to
$66 \%$ for $\$$-to- $\$$ misses, $45 \%$ for $M e m$ misses, $45 \%$ for Inv misses and $55 \%$ for Inv+Mem misses have been obtained. These reductions translate into important improvements in the application execution times (reductions up to $34 \%$ ).

\section{Acknowledgments}

This research has been carried out using the resources of the Centre de Computació i Comunicacions de Catalunya (CESCA-CEPBA) as well as the SGI Origin 2000 of the Universitat de Valencia. This work has been supported in part by the Spanish CICYT (grant TIC2000-1151-C07).

\section{References}

[1] M. E. Acacio, J. González, J. M. García and J. Duato. "A New Scalable Directory Architecture for Large-Scale Multiprocessors". 7th Int'l Symposium on High Performance Computer Architecture, Jan. 2001.

[2] M. E. Acacio, J. González, J. M. García and J. Duato. "A Novel Approach to Reduce L2 Miss Latency in SharedMemory Multiprocessors". Tech. Report UM-DITEC-20021 , Computer Engineering Department, University of Murcia, Jan. 2002

[3] L. A. Barroso, K. Gharachorloo and E. Bugnion. "Memory System Characterization of Commercial Workloads". 25th Int'l Symposium on Computer Architecture, June 1998.

[4] L. A. Barroso, K. Gharachorloo, R. McNamara, A. Nowatzky, S. Qadeer, B. Sano, S. Smith, R. Stets and B. Verghese. "Piranha: A Scalable Architecture Based on Single-Chip Multiprocessing". 27th Int'l Symposium on Computer Architecture, June 2000.

[5] D. E. Culler, J. P. Singh and A. Gupta. "Parallel Computer Architecture: A Hardware/Software Approach”. Morgan Kaufmann Publishers, Inc., 1999.

[6] A. Gupta, W.-D. Weber and T. Mowry. "Reducing Memory and Traffic Requirements for Scalable Directory-Based Cache Coherence Schemes". Int'l Conference on Parallel Processing, August 1990

[7] L. Gwennap. "Alpha 21364 to Ease Memory Bottleneck". Microprocessor Report, pp. 12-15, October 1998.

[8] L. Hammond, M. Willey and K. Olukotun. "The Standford Hydra CMP”. Proc. of Hot Chips 11, August 1999.

[9] J. Kuskin, D. Ofelt, M. Heinrich, J. Heinlein, R. Simoni, K. Gharachorloo, J. Chapin, D. Nakahira, J. Baxter, M. Horowitz, A. Gupta, M. Rosenblum and J. Hennessy. "The Stanford FLASH Multiprocessor". 21st Int'l Symposium on Computer Architecture, Apr. 1994.

[10] J. Laudon and D. Lenoski. "The SGI Origin: A ccNUMA Highly Scalable Server". 24th Int'l Symposium on Computer Architecture, June 1997.

[11] T. Lovett and R. Clapp. "STiNG: A CC-NUMA Computer System for the Commercial Marketplace". 23rd Int'l Symposium on Computer Architecture, 1997.

[12] M. M. Michael and A. K. Nanda. "Design and Performance of Directory Caches for Scalable Shared Memory Multiprocessors". 5th Int'l Symposium on High Performance Computer Architecture, Jan. 1999.

[13] B. O'Krafka and A. Newton. "An Empirical Evaluation of Two Memory-Efficient Directory Methods". 17th Int'l Symposium on Computer Architecture, May 1990.

[14] V. Pai, P. Ranganathan and S. Adve. "RSIM Reference Manual version 1.0". Tech. Report 9705, Department of Electrical and Computer Engineering, Rice University, Aug. 1997.

[15] J. Torrellas, L. Yang and A. T. Nguyen. "Toward A Cost-Effective DSM Organization That Exploits ProcessorMemory Integration". 6th Int'l Symposium on High Performance Computer Architecture, Jan. 2000.

[16] S. C. Woo, M. Ohara, E. Torrie, J. P. Singh and A. Gupta. "The SPLASH-2 Programs: Characterization and Methodological Considerations". 22nd Int'l Symposium on Computer Architecture, June 1995. 